\title{
Factors associated with the misdiagnosis of sentinel lymph nodes using touch imprint cytology for early stage breast cancer
}

\author{
YI-ZUO CHEN $^{1,4^{*}}$, JIA-XIN ZHANG ${ }^{1 *}$, JIA-JIAN CHEN ${ }^{1}$, ZHE-BIN LIU $^{1}$, XIAO-YAN HUANG ${ }^{1}$, \\ JING YI CHENG ${ }^{2}$, WEN-TAO YANG ${ }^{3}$, ZHI-MIN SHAO ${ }^{1}$, ZHEN-ZHOU SHEN $^{1}$ and JIONG WU ${ }^{1}$
}

\author{
Departments of ${ }^{1}$ Breast Surgery, ${ }^{2}$ Nuclear Medicine, and ${ }^{3}$ Pathology, Fudan University Shanghai Cancer Center, \\ Shanghai 200032; ${ }^{4}$ Department of Oncology Surgery, The First Affiliated Hospital of \\ Wenzhou Medical College, Wenzhou, Zhejiang 325000, P.R. China
}

Received August 3, 2010; Accepted January 3, 2011

DOI: $10.3892 / 01.2011 .233$

\begin{abstract}
Accurate intraoperative diagnosis of sentinel node metastasis enables the surgeon to make an immediate decision to proceed to axillary lymph node dissection (ALND), thereby avoiding the economic and psychological costs of a second operation. The present study aimed to evaluate the clinical value of touch imprint cytology (TIC) and investigate the potential factors associated with misdiagnosis. A total of 366 patients with Tis-T2 breast carcinoma were included after undergoing successful sentinel lymph node biopsy (SLNB). TIC was routinely performed intraoperatively, and the results were compared with definitive histological assessments of serial sections (SS) with hematoxylin and eosin (H\&E) staining. A total of 992 SLNs from 366 patients were used in the study. Based on the final histological diagnosis, the sensitivity, specificity and overall accuracy of TIC was 76.6, 98.8 and $92.3 \%$, respectively, on a per patient basis, and 79.9, 98.9 and $96.1 \%$, respectively, on a per node basis. TIC was significantly more sensitive for macrometastasis than micrometastasis $(80.0$ vs. $28.6 \%, \mathrm{P}<0.01)$. Of 9 total 'false positives', 3 were due to micrometastasis which were not identified by serial section with H\&E staining, 4 were actual false-positives which were due to interpretation error, and 2 were due to sampling error. The majority of the false-negatives (28 of 30 SLNs) were due to micrometastasis in the SLNs (sampling error). In conclusion, TIC is feasible for clinical use and is able to detect macrometastasis in the SLNs of early stage invasive breast cancer patients with an acceptable accuracy while its ability to detect micrometastasis is limited.
\end{abstract}

Correspondence to: Dr Jiong Wu, Department of Breast Surgery, Fudan University Shanghai Cancer Center, 270 Dong An Road, Shanghai 200032, P.R. China

E-mail:wujiong1122@vip.sina.com

${ }^{*}$ Contributed equally

Key words: breast carcinoma, sentinel node biopsy, intraoperative diagnosis, touch imprint cytology, micrometastasis

\section{Introduction}

Sentinel lymph node biopsy (SLNB) is emerging as the new standard for axillary staging in early breast cancer, supported by a number of randomized controlled trials (1). Intraoperative detection of sentinel node metastases enables the surgeon to make an immediate decision to proceed to axillary lymph node dissection (ALND), thereby avoiding the economic and psychological costs of a second operation. However, in order to implement the procedure in clinical practice, a method for accurate intraoperative analysis of the SLNs is required in order that the decision to avoid ALND can be made during primary surgery in the case of negative SLNs. This process can be achieved by means of frozen sectioning (FS) and touch imprint cytology (TIC). Results of numerous studies showed that TIC has a sensitivity equivalent to or even better than that of FS (2-5), since TIC offers the advantages of minimal tissue preparation, rapid staining and good cytological detail for interpretation. No special equipment is required and no tissue loss occurs (6). Therefore, it is recommended by the College of American Pathologists that TIC be applied in the intraoperative evaluation of SLNs (7).

A large series of related studies focused on the evaluation of the sensitivity of TIC and reviewed the risk factors associated with false-negative cases which may lead to a secondary ALND. However, few studies have focused on false-positive cases which result in an unnecessary ALND and potential medicolegal repercussions that discourage both surgeons and patients (2).

The primary aim of the present study was to evaluate the clinical value of TIC with a normative procedure of the pathological evaluation of SLNs. The secondary aim was to investigate the potential factors associated with the misdiagnosed results.

\section{Materials and methods}

Patients. A consecutive series of 366 women with T1-T2 invasive breast cancer treated at the Department of Breast Surgery, Cancer Hospital, Fudan University, China, between February 2005 and March 2008, participated in our previous study on SLNB. Eligibility criteria included i) diagnosis 
of operable primary breast cancer $\leq 5 \mathrm{~cm}$ in diameter and a unicentric lump using clinical and imaging criteria confirmed by core or open biopsy; ii) confirmation of negative axillary lymph nodes under clinical and imaging examination; iii) confirmation of no previous surgery performed in the axilla iv) non-pregnant status v) obtainment of informed consent; and vi) the harvesting of at least one SLN during the surgery. Certain relatively rare types of breast carcinoma were excluded in order that the focus remain on the three most common types of breast carcinoma: invasive ductal carcinoma (IDC), ductal carcinoma in situ (DCIS), and ductal carcinoma in situ with microinvasion (DCIS-Mi).

TIC procedure and pathological evaluation. Details on the SLNB procedure at our institution were previously reported (8). The SLNs were cut along the long axis at a 2.0- to $3.0-\mathrm{mm}$ interval intraoperatively, and each cut surface was touched at least three times onto a clean glass slide and stained with hematoxylin and eosin (H\&E). The slides were sent to cytopathologists immediately after the slides were prepared. The results obtained from the cytopathologists were used by the surgeon as the primary intraoperative tool for determining whether ALND should be performed. Slices were formalinfixed and paraffin-embedded for further evaluation.

Definitive histological assessment was performed on the paraffin-embedded tissue. Serial sections (SS) at a $100-\mu \mathrm{m}$ interval with standard $H \& E$ staining were carried out postoperatively. S-P immunohistochemical (IHC) staining (3- to 5- $\mu \mathrm{m}$ thick) with CK-19 (clone EP1580Y; Epitomics, Burlingame, CA) and MUC-1 (clone Ma695; Novo-Castra, Newcastle, UK) was performed unless macrometastasis was indicated with H\&E staining. The pathological results were classified as macrometastasis $(>2.0 \mathrm{~mm})$, micrometastasis $(0.2-2.0 \mathrm{~mm})$ and isolate tumor cells (ITC, $<0.2 \mathrm{~mm}$ ) according to the TMN staging system (9). Patients with negative TIC but positive for either SS with H\&E staining or IHC (except for micrometastasis or ITC with IHC) required a second axillary operation.

Statistical analysis. The results of TIC were compared with those of the final pathology and were classified as true-positive (TP), true-negative (TN), false-negative (FN) or false-positive (FP), both on a patient and node basis. True-positive cases were those that were found to contain carcinoma both on TIC and on subsequent H\&E and IHC staining. The formulas used to calculate statistical parameters were: Sensitivity $=\mathrm{TP} /(\mathrm{TP}$ $+\mathrm{FN})$; specificity $=\mathrm{TN} /(\mathrm{TN}+\mathrm{FP})$; overall accuracy $=(\mathrm{TP}+$ $\mathrm{TN}) /(\mathrm{TP}+\mathrm{FP}+\mathrm{TN}+\mathrm{FN})$; negative predictive value $(\mathrm{NPV})=$ $\mathrm{TN} /(\mathrm{TN}+\mathrm{FN})$ and positive predictive value $(\mathrm{PPV})=\mathrm{TP} /(\mathrm{TP}+$ FP). For statistical analysis, Fisher's exact test was performed with a cut-off point of $\mathrm{P}<0.05$ using the SPSS statistical analysis program, version 16.0 (SPSS Inc., Chicago, IL, USA).

\section{Results}

Pricipal observations. The mean age of the 366 patients was 49.9 years (range 24-81). The median size of the measured primary tumors was $22 \mathrm{~mm}(4-5 \mathrm{~mm})$. The mean number of the SLNs was 2.75 per patient. For all surgeries, the results of the intraoperative assessment of the SLNs were available prior to completion of the lumpectomy or mastectomy.
Table I. The total sensitivity, specificity, overall accuracy rate, negative and positive predictive value of touch imprint cytology for the studied cases.

Final histopathological diagnosis ${ }^{\mathrm{a}}$

\begin{tabular}{|c|c|c|}
\hline TIC & By case & By node \\
\hline Sensitivity & $76.6 \% \quad(82 / 107)$ & $79.9 \%(119 / 149)$ \\
\hline Specificity & $98.8 \%(256 / 259)$ & $98.9 \%(834 / 843)$ \\
\hline Overall accuracy & $92.3 \%(338 / 366)$ & $96.1 \%(953 / 992)$ \\
\hline NPV & $91.1 \%(256 / 281)$ & $96.5 \%(834 / 864)$ \\
\hline PPV & $96.5 \% \quad(82 / 85)$ & $93.0 \%(119 / 128)$ \\
\hline
\end{tabular}

aUsing serial sections with $\mathrm{H} \& \mathrm{E}$ as the final histopathologic diagnosis. TIC, touch imprint cytology; NPV, negative predictive value; PPV, positive predictive value.

Table II. Analysis of touch imprint cytology (TIC) of the metastatic deposit with regard to its size.

\begin{tabular}{lccc}
\hline $\begin{array}{l}\text { SLN metastasis size at } \\
\text { final histopathology }\end{array}$ & TIC & $\mathrm{n}$ & Sensitivity (\%) \\
\hline Macrometastases & Positive & 80 & $80.0 \%$ \\
$(\mathrm{n}=100)$ & Negative & 20 & \\
Micrometastases or & Positive & 2 & $28.6 \%$ \\
ITC (n=7) & Negative & $5^{\mathrm{a}}$ & \\
No. of metastases & Positive & 3 & \\
$(\mathrm{n}=259)$ & Negative & 256 & \\
\hline
\end{tabular}

ancluding one patient with isolate tumor cells (ITC). TIC, touch imprint cytology; SLN, sentinel lymph nodes.

Based on the final pathology, among the 107 cases in which positive lymph nodes were present, 74 (69.2\%) had at least one sentinel lymph node that was positive. Overall, 149 positive lymph nodes were removed. TIC identified 82/107 patients and a total 119/149 nodes, as well as 3 false-positive patients ( 9 nodes), resulting in a sensitivity, specificity and overall accuracy rate of $76.6,98.8$ and $92.3 \%$, respectively, on a per patient basis, and 79.9, 98.9 and $96.1 \%$, respectively, on a per node basis (Table I). Micrometastases were found in 19 SLNs, of which 6 nodes were identified by TIC. Only 7/107 (6.5\%) patients had SLNs that were positive for micrometastasis but no macrometastasis in any other SLNs, and 5/7 were overlooked by TIC, resulting in a sensitivity for micrometastasis of $28.6 \%$ on a per patient basis and $31.6 \%$ on a per node basis. Sensitivity for macrometastasis was $80.0 \%$ on a per patient basis and $86.9 \%$ on a per node basis (Table II).

Metastatic foci of 83/107 (77.6\%) patients were identified in the initial SLN captured. Additionally, all of the patients that had metastatic SLNs were confirmed to be node involved within the first four SLNs harvested.

Among the 366 patients, the postoperative pathological evaluation determined DCIS in 33 patients, DCIS-Mi in 25 patients and IDC in 308 patients. The metastatic rates of 


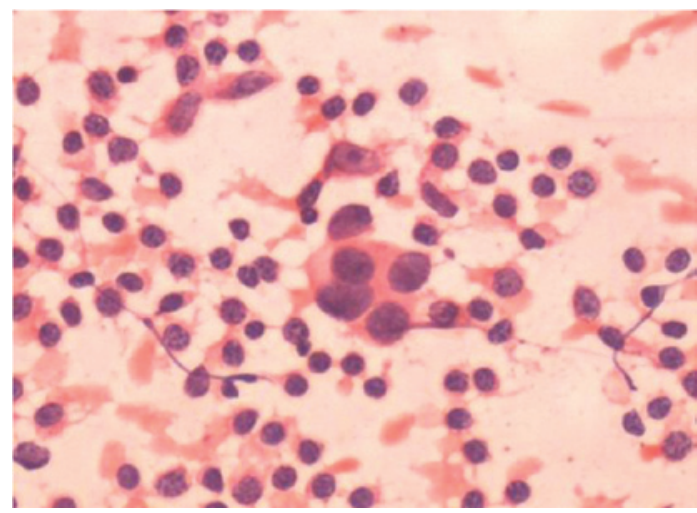

Figure 1. An atypical node thought to be positive intraoperatively. (H\&E; magnification, $\mathrm{x} 200)$.

the patients with DCIS, DCIS-Mi and IDC were 3.0\% (1 case), $16.0 \%$ (4 cases) and $33.1 \%$ (102 cases), respectively. For the patients with DCIS, DCIS-Mi and IDC, 1.0\% (1/97 nodes), $10.0 \%$ (6/60 nodes) and 34.1\% (285/835 nodes) SLNs captured, respectively, were confirmed to contain cancer cells. The sensitivity of TIC for patients with DCIS, DCIS-Mi and IDC was $0,50.0$ and $78.4 \%$, respectively, on a per patient basis, and $0,66.7$ and $90.5 \%$, respectively, on a per node basis.

False-positive results based on serial sections with hemato-xylin and eosin. A total of 9 SLNs from 3 patients were positive upon TIC and negative using SS with H\&E staining; the 3 patients received an unnecessary ALND. Four of the 9 'false-positive' nodes were considered to be atypical when reviewed (Fig. 1). Subsequent IHC staining identified three micrometastasispositive SLNs (Fig. 2). On reviewing the remaining 2 'false positive' SLNs, malignant cells were found in the two cases that were considered to be positive by the cytopathologists.

False-negative results based on serial sections with hematoxylin and eosin staining. A total of 30 SLNs from 25 patients were false-negative, and micrometastases were detected in 13/30 (43.3\%) SLNs based on SS with H\&E staining. The result of the IHC staining showed 2 micrometastasis-positive SLNs using SS with H\&E staining that were assessed to be macrometastatic. The false-negatives were examined to determine whether the misdiagnoses were due to interpretation or sampling error. Two imprints showed cytological evidence of metastases (interpretation error), whereas the remaining 28 imprints were due to sampling error. All of the 25 falsenegative patients underwent a second axillary operation.

\section{Discussion}

The results from the present study are in accordance with previously published data indicating that the TIC technique provides acceptable accuracy in detecting macrometastasis, but not micrometastasis $(2,6,10,11)$. The sensitivity of TIC in published studies varies widely from $34 \%$ (11) to $96 \%$ (9). The sensitivity in our study was moderate $(76.6 \%)$ by taking $2-$ to $3-\mathrm{mm}$ serial slices of the sentinel node. Studies have found that this cutting interval may increase the sensitivity of TIC by increasing the effective surface area for analysis, allowing for the detection of small deposits and micrometastasis while increasing the pathological reporting time $(2,11-16)$. In our institution, intraoperative assessment is accomplished during primary surgery and does not appear to prolong the surgical procedure.

It should be noted that the metastastic rate of patients is as low as $3.0 \%$ for patients with DCIS, whereas the sensitivity for TIC in patients with DCIS is 0 and $50 \%$ for DCIS-Mi patients. Despite these results, intraoperative evaluation in patients with DCIS or DCIS-Mi diagnosed by core needle biopsy was performed, since these preoperative histopathological assessments did not reach a high enough specificity to accurately diagnose the majority of the pure DCIS cases. Ultrasoundguided core needle biopsy is considered to be a reliable non-invasive alternative to surgical biopsy for obtaining a histopathological diagnosis with considerable sensitivity and specificity. However, its limitations include relatively high false-negative results (range 0.9-9.0\%) and underestimation of disease with a wide range (17), suggesting that it is necessary to perform intraoperative assessment for patients with DCIS diagnosed by core needle biopsy who may need SLNB.
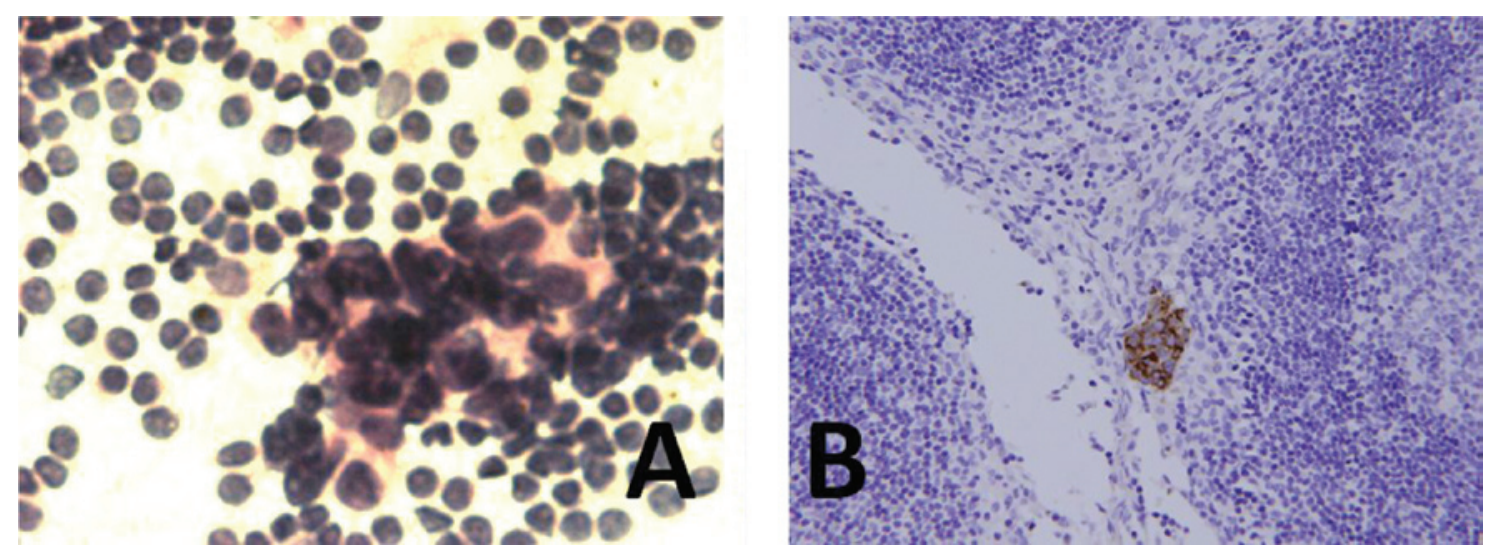

Figure 2. A 'false-positive' sentinel lymph node that was positive with both touch imprint cytology and immunohistochemistry (IHC) but negative with H\&E staining. (A) Positive touch imprint cytology of a sentinel lymph node involved with cancer cells (H\&E; magnification, $\mathrm{x} 400$ ). (B) IHC staining for cytokeratin for the same sentinel lymph node as in (A) (IHC for CK-19; magnification, x200). 
Studies have indicated that lobular histology may be more difficult to interpret using touch imprint cytology, leading to a higher false-negative rate (18). Lobular carcinomas were excluded in our study due to the limited number of cases (4 cases), and we focused on the ductal carcinoma type. We found 25 false-negative and 3 false-positive patients using TIC, and aimed to identify the potential and common factors associated with the misdiagnosed results.

The findings showed 9 false-positive SLNs in our series. The 9 SLNs were re-examined, and positive results for the 5 imprints were confirmed following revision; 3 of the 5 were identified as micrometastasis-positive SLNs by subsequent IHC staining. Four of the 9 false-positive nodes were considered to be atypical when reviewed. Three possible reasons exist for the false-positive result. Firstly, micrometastatic foci which were overlooked by SS with H\&E may have been detected by TIC. This is rare and cases should be considered true positive when subsequent IHC staining indicates micrometastasis. Therefore, for the false-positive cases based on SS with H\&E staining, SS with IHC staining should be performed to avoid misdiagnosis. Secondly, the imprint cytology specimen may have contained the only metastatic deposit in the part of the lymph node that was lost in the deeper sections of the lymph node and, consequently, was not located on final histopathology. Finally, the clinical impression of the surgeon regarding the appearance of the nodes may affect the decision of the cytopathologists. Cytopathologists may be required to examine the nodes thoroughly when the nodes are pathologically palpable or the cut surfaces of the SLNs are suspicious. This requirement may lead to cytopathologists being overly cautious when deciding on a negative result when the imprints appear to be atypical. In the present study, the surgeon's clinical impression was that 4 false-positive SLNs, using SS with H\&E staining, were atypical. However, these SLNs were determined to be carcinoma cells by the cytopathologists.

In the present study, 30 false-negative SLNs were found, and micrometastasis was detected in 13/30 (43.3\%) SLNs, using SS with H\&E staining. We conclude that the main reason for the false-negative results of the imprint cytology was poor quality of the imprint samples due to sampling error. One of the significant factors impacting sampling error is micrometastasis in sentinel nodes. SLNs were cut along the long axis at a 2.0 - to $3.0-\mathrm{mm}$ interval to prepare imprints while SLNs were cut at a $100-\mu \mathrm{m}$ interval to perform the final histopathological diagnosis. Therefore, small metastatic foci may be not be detected by TIC but identified by final histopathological diagnosis due to the more precise cutting method. The intraoperative detection of micrometastatic disease is a well-known issue, leading to high false-negative rates. A metaanalysis (19) of 31 related studies found a pooled sensitivity of $63 \%$ (95\% CI, 57-69) and specificity of 99\% (95\% CI, 98-99) for TIC; the pooled sensitivity for macrometastasis was $81 \%$ and that for micrometastasis was $22 \%$. The only statistically significant predictor for a false-negative evaluation of axillary node involvement was found to be the proportion of micrometastasis in multivariable analysis (19). In the present study, TIC was significantly more sensitive for macrometastasis than micrometastasis; 80.0 vs. $28.6 \%$, respectively, $(\mathrm{P}=0.001)$. It has been suggested that shortening the cutting interval may be useful for improving the quality of the imprint samples.
Therefore, a prospective controlled study commenced in March 2008 at our institution to compare the clinical value of the cutting method in preparing imprints between the routine protocol (to cut along the long axis at a 2.0 - to $3.0-\mathrm{mm}$ interval) and the new protocol (to cut along the short axis at a $1.5-\mathrm{mm}$ interval).

In conclusion, at our institution, TIC is considered an accurate, practical, time- and cost-efficient procedure with minimal tissue preparation for intraoperatively evaluating SLNs. It is feasible for clinical use and is able to detect macrometastasis in SLNs intraoperatively with an acceptable accuracy early stage invasive breast cancer patients, although its ability to detect micrometastasis is limited. Furthermore, micrometastasis in SLNs and interpretation error may be the key reasons for false-positive and false-negative results in the intraoperative evaluation of SLNs.

\section{Acknowledgements}

The authors thank the patients and family members for their willingness to cooperate in our study. Grants were endowed by the Research Foundation of Sci-Tech Committee of Shanghai City (no. 54119524) and the 'Shu Guang' Project Foundation of the Education Committee of Shanghai City (no. 05SG04).

\section{References}

1. Veronesi U, Paganelli G, Viale G, et al: A randomized comparison of sentinel-node biopsy with routine axillary dissection in breast cancer. N Engl J Med 349: 546-553, 2003.

2. Creager AJ, Geisinger KR, Shiver SA, et al: Intraoperative evaluation of sentinel lymph nodes for metastatic breast carcinoma by imprint cytology. Mod Pathol 15: 1140-1147, 2002.

3. Liang R, Craik J, Juhasz ES and Harman CR: Imprint cytology versus frozen section: intraoperative analysis of sentinel lymph nodes in breast cancer. ANZ J Surg 73: 597-599, 2003.

4. Aihara T, Munakata S, Morino H and Takatsuka Y: Comparison of frozen section and touch imprint cytology for evaluation of sentinel lymph node metastasis in breast cancer. Ann Surg Oncol 11: 747-750, 2004.

5. Motomura K, Inaji H, Komoike Y, et al: Intraoperative sentinel lymph node examination by imprint cytology and frozen sectioning during breast surgery. Br J Surg 87: 597-601, 2000.

6. Creager AJ, Geisinger KR, Perrier ND, et al: Intraoperative imprint cytologic evaluation of sentinel lymph nodes for lobular carcinoma of the breast. Ann Surg 239: 61-66, 2004.

7. Fitzgibbons PL, Page DL, Weaver D, et al: Prognostic factors in breast cancer. College of American Pathologists Consensus Statement 1999. Arch Pathol Lab Med 124: 966-978, 2000.

8. Chen JJ, Huang XY, Liu ZB, et al: Sentinel node biopsy and quality of life measures in a Chinese population. Eur J Surg Oncol 35: 921-927, 2009.

9. Rubio IT, Korourian S, Cowan C, Krag DN, Colvert M and Klimberg VS: Use of touch preps for intraoperative diagnosis of sentinel lymph node metastases in breast cancer. Ann Surg Oncol 5: 689-694, 1998.

10. Menes TS, Tartter PI, Mizrachi H, et al: Touch preparation or frozen section for intraoperative detection of sentinel lymph node metastases from breast cancer. Ann Surg Oncol 10: 1166-1170, 2003.

11. Zgajnar J, Frkovic-Grazio S, Besic N, et al: Low sensitivity of the touch imprint cytology of the sentinel lymph node in breast cancer patients - results of a large series. J Surg Oncol 85: 82-86, discussion 8, 2004.

12. Mullenix PS, Carter PL, Martin MJ, et al: Predictive value of intra-operative touch preparation analysis of sentinel lymph nodes for axillary metastasis in breast cancer. Am J Surg 185: 420-424, 2003. 
13. Bochner MA, Farshid G, Dodd TJ, Kollias J and Gill PG: Intraoperative imprint cytologic assessment of the sentinel node for early breast cancer. World J Surg 27: 430-432, 2003.

14. Karamlou T, Johnson NM, Chan B, Franzini D and Mahin D: Accuracy of intraoperative touch imprint cytologic analysis of sentinel lymph nodes in breast cancer. Am J Surg 185: 425-428, 2003.

15. Baitchev G, Gortchev G and Todorova A: Intraoperative sentinel lymph node examination by imprint cytology during breast surgery. Curr Med Res Opin 18: 185-187, 2002.

16. Llatjos M, Castella E, Fraile M, et al: Intraoperative assessment of sentinel lymph nodes in patients with breast carcinoma: accuracy of rapid imprint cytology compared with definitive histologic workup. Cancer 96: 150-156, 2002.
17. Schueller G, Schueller-Weidekamm C and Helbich TH: Accuracy of ultrasound-guided, large-core needle breast biopsy. Eur Radiol 18: 1761-1773, 2008

18. Cox C, Centeno B, Dickson D, et al: Accuracy of intraoperative imprint cytology for sentinel lymph node evaluation in the treatment of breast carcinoma. Cancer 105: 13-20, 2005.

19. Tew K, Irwig L, Matthews A, Crowe $P$ and Macaskill P: Metaanalysis of sentinel node imprint cytology in breast cancer. Br J Surg 92: 1068-1080, 2005. 